\title{
A case of the syndrome of inappropriate antidiuretic hormone secretion treated successfully with Tolvaptan to prevent hospital admission and delay chemotherapy treatment in patient with a small cell lung cancer
}

Dr Kamal Abouglila, Department of Endocrinology University Hospital North Durham

\section{BACKGROUND}

Hyponatraemia is the most commonly recorded electrolyte abnormality occurring in $7 \%$ to $8 \%$ of elderly, ambulatory patients and 15 to $20 \%$ of hospitalized patients presenting with a variety of symptoms ranging from very mild to life threatening. Correction of hyponatremia has been shown to improve the symptoms and signs associated with this condition. It is also recognized that excessively rapid correction of hyponatremia can be detrimental. During hospital admission, hyponatremia is also associated with increased length of stay and worse primary clinical outcomes. Fluid restriction remains the mainstay of treatment for moderate hyponatremia associated with SIADH. However, fluid restriction is often challenging and unpleasant for patients and when therapies such as chemotherapy for malignant disease are planned it is not a preferred option due to risk of dehydration and acute kidney injury.

\section{CASE STUDY}

We describe a Case of 71 years male who presented with recurrent admission with severe hyponatremia (SIADH) due to metastatic small cell cancer which failed to response to standard treatment of SIADH including using demeclocyline therapy and as a sequence to failure of correcting hyponatremia his chemotherapy treatment has been postponed in a few occasions.

\section{DISCUSSION}

We decided to treat his hyponatremia by using Tolvaptan which it is antagonism at the $\mathrm{V} 2$ receptor causes a decrease in the number of aquaporin-2 channels in the renal collecting tubules, resulting in decreased water reabsorption, a net increase in free water excretion, and an increase in serum sodium concentrations. Sodium improved with a few days and he had not a further admission with similar problems and he had his chemotherapy treatment for the lung cancer.

\section{Conclusion:}

This case highlights the importance of the use of Tolvaptan in patients with recurrent admission with hyponatremia and it patients who are chemotherapy postponed. It is help to reduce the length of Hospital stay.

\section{REFERENCES}

Castillo JJ1 , Oncologist. 2012;17(6):756-65-Diagnosis and management of hyponatremia in cancer patients. 